\title{
Participación en medios y TIC: intereses y expectativas de niños y níñas
}

\author{
Andrea Lafaurie-Molina, Ph.D. ${ }^{a}$ \\ Universidad del Norte, Colombia \\ Rocío López-Ordosgoitia, Ph.D.b \\ Pontificia Universidad Javeriana, Colombia
}

\author{
Diana Alexandra Giraldo-Cadavid, Ph.D. ${ }^{c}$ \\ Universidad del Valle, Colombia \\ Diana Marcela Aristizábal-García, Ph.D. ${ }^{d}$ \\ Universidad de San Buenaventura, Colombia
}

andreal@uninorte.edu.co

\section{Resumen (analítico)}

La presencia de medios análogos y digitales en la vida de los niños, niñas y adolescentes redimensiona la discusión sobre participación infantil, centrándola en lo que ocurre en las pantallas. Realizamos un estudio cualitativo de corte descriptivo con 75 niños, niñas y adolescentes, de 7 a 14 años, de tres ciudades (Barranquilla, Cali y Bogotá). Los resultados indican que los niños, niñas y adolescentes tienen formas complejas y diversas de pensar la participación, que superan las divisiones, escalas y prescripciones adultas e institucionales, para incluir acciones de ocio, socialización y consumo cultural. Se trata de una participación heterogénea, convergente y multiplataforma que incluye actividades como generar contenidos, dar likes, hacer suscripciones, navegar, buscar tareas o simplemente ver vídeos, fotos, jugar, divertirse y socializar, mostrando intereses, subjetividades y formas de socialización infantiles en medios y TIC.

\section{Palabras clave}

Participación infantil, TIC, infancia, plataformas virtuales, interacción virtual, comunicación virtual, ecosistema mediático, participación convergente, participación multiplataforma.

\section{Thesauro}

Tesauro de Ciencias Sociales de la Unesco.

\section{Para citar este artículo}

Lafaurie-Molina, A., López-Ordosgoitia, R., GiraldoCadavid, D. A., \& Aristizábal-García, D. M. (2022). Participación en medios y TIC: intereses y expectativas de niños y niñas. Revista Latinoamericana de Ciencias Sociales, Niñez y Juventud, 20(1), 1-22. https://dx.doi.org/10.11600/rlcsnj.20.1.4934

\section{Historial}

Recibido: 03.06.2021

Aceptado: 07.10.2021

Publicado: 03.12.2021

\section{Información artículo}

Los resultados de este artículo hacen parte de la investigación titulada Think Big Colombia. ¿Y si hablamos de participación en los medios? Un estudio desde la perspectiva de los niños y niñas colombianos, desarrollada por la Universidad del Norte, Pontificia Universidad Javeriana y Universidad del Valle. Su primera fase fue iniciada en septiembre de 2018 y finalizada en diciembre de 2020. Hace parte de la iniciativa global Think Big, liderada por la Universidad de Melbourne, con financiación de VicHealth. Área: humanidades y ciencias sociales. Subárea: comunicación y medios. 


\section{Participation in media and ICTs: children's interests and expectations}

\section{Abstract (analytical)}

The presence of analogue and digital media in the lives of children and adolescents reshapes discussions on child participation, with an emphasis on participation that is facilitated through screens. A qualitative descriptive study was carried out with 75 children and adolescents aged 7 to 14 years old in three cities (Barranquilla, Cali and Bogotá). The results suggest that children and adolescents have complex and diverse ways of thinking about participation that go beyond adult-centric and institutional concepts such as divisions and different scales. Children conceive their own participation in terms of leisure, socialization and cultural consumption activities. Theirs is a heterogeneous, convergent and multi-platform participation that includes activities such as creating content, liking, subscribing, browsing the web, doing homework, as well as watching videos, looking at pictures, playing games, having fun and socializing. These results show children's interests, subjectivities and forms of socialization using media and ICTs.

\section{Keywords}

Child participation, ICT, children, virtual platforms, virtual interaction, virtual communication, media ecosystem, convergent participation, multiplatform participation.

\section{Participação nos meios e nas TIC: os interesses e as expectativas das crianças}

\section{Resumo (analítico)}

A presença da mídia analógica e digital na vida das crianças e adolescentes, redimensiona a discussão sobre a participação infantil enfocando o que acontece nas telas. Foi realizado um estudo qualitativo descritivo com 75 crianças de 7 a 14 anos em três cidades (Barranquilla, Cali e Bogotá). Os resultados indicam que as crianças e adolescentes têm formas complexas e diversas de pensar a participação, assim como seus papéis na mídia e no cenário digital, que vão além das divisões, escalas e prescrições de adultos e instituições. Esta participação é heterogênea, convergente e multiplataforma, que inclui atividades como a criação de conteúdo, dar likes, fazer trabalhos escolares ou simplesmente olhar vídeos, fotos, jogar, brincar e socializar; mostrando os interesses, subjetividades e formas de socialização das crianças na mídia e nas TIC.

\section{Palavras-chave}

Participação das crianças, TIC, infancia, plataformas virtuais, interação virtual, comunicação virtual, ecossistema de mídia, participação convergente, participação multiplataforma.

\section{Información autoras}

[a] Comunicadora Social y Periodista. Magíster en Comunicación y Educación, Universidad Autónoma de Barcelona. Doctora en Contenidos de comunicación en la Era Digital, Universidad Autónoma de Barcelona. Orcid: 0000-0002-6686-2194. H5: 5. Correo electrónico: andreal@uninorte.edu.co

[b] Comunicadora Social y Periodista. Maestría en Ingeniería de Medios para la Educación, Universidad de Poitiers y Universidad de Lisboa. Doctora en Ciencias de la Información y la Comunicación, Universidad de Lille. Orcid: 0000-0002-5937-941X. H5: 1. Correo electrónico: rvlopez@javeriana.edu.co

[c] Comunicadora Social y Periodista. Maestría en Filosofía, Universidad del Valle. Doctora en Ciencias de la Información y la Comunicación, Universidad Lyon 2. Orcid: 0000-0003-2154-9425. H5: 5. Correo electrónico:

diana.a.giraldo@correounivalle.edu.co

[d] Periodista e historiadora. Maestría en Estudios Culturales, Universidad de los Andes. Doctora en Antropología Social, Universidad de Los Andes. Orcid: 0000-0003-1709-9986. H5: 2. Correo electrónico: dianaaristizabal@usantotomas.edu.co 


\section{Introducción}

Con la declaración de la Convención de los derechos del niño (ONU, 1989) la discusión política y académica sobre la participación infantil se intensificó, sobre todo en contextos occidentales. Desde entonces, esta se considera un indicador para determinar hasta qué punto los países toman en cuenta a los niños, niñas y adolescentes (en adelante NNA) como sujetos de derecho y cuál es el lugar que estos pueden tener sobre sus propias vidas y el devenir de sus comunidades. La literatura académica sobre la participación infantil se ha enfocado sobre todo en problematizar esta noción, mostrando que, aunque generalmente se asocia, primero, a un derecho y, segundo, a una actividad con una connotación positiva, la misma puede tener muchas dimensiones, valoraciones morales, grados de acción e, incluso, limitaciones de contexto para llevarla a cabo (Cuevas-Parra, 2021). Puede ser activa, pasiva, voluntaria, forzada, organizada, espontánea, simbólica o manipulada; puede responder a diferentes áreas de la vida (económica, política, social, cultural), así como a objetivos individuales o colectivos, y debe hacer parte de la vida de los NNA, pertenezcan o no a los marcos jurídicos de los Estados que firmaron la Convención (Gaitán \& Liebel, 2011; Liebel \& Markowska-Manista, 2021).

Así, las reflexiones teóricas y epistemológicas sobre esta discusión se han nutrido de diferentes campos de conocimiento, autores y preguntas. Hart (1993) fue uno de los primeros en presentar una definición sobre la participación de los NNA, la cual abarca «los procesos de compartir las decisiones que afectan la vida propia y la vida en la comunidad en la que se vive» (p. 5). A partir de este postulado, otros autores (Cornwall, 20o8; Kay et al., 2021; Lundy, 2018; Trilla \& Novella, 2001) propusieron diferentes clasificaciones teóricas sobre los tipos o niveles de participación infantil, y han diferenciado entre la concepción instrumental y utilitarista de esta (como medio) y la que resulta transformadora, liberadora y constructora de ciudadanía en un sentido amplio (como objetivo).

A esta tradición se han sumado, desde una perspectiva crítica y decolonial, las reflexiones de Liebel y Saadi (2012), quienes invitan a superar la idea occidental e individualista 
(liberal) de la participación. Los autores proponen pensarla como un asunto de carácter relacional entre NNA y adultos, intentando visualizar la manera en que la participación infantil es comprendida en diferentes culturas y sociedades que, a la vez, tienen ideas diversas sobre la infancia como etapa de la vida y sobre los roles sociales de los NNA. Liebel problematiza, además, la noción de protagonismo infantil, concepto usado por varios autores (Lay-Lisboa \& Montañes, 2018; Salazar-Guizzo et al., 2019; Soares Gouvêa et al., 2019), que «pone a los niños en el centro de la sociedad y les confía la fuerza y la capacidad de tener un papel esencial» (Liebel, 2007, p. 114).

Adicionalmente, se puede identificar una tercera tendencia enfocada en hacer una crítica a los alcances de la Convención y a las políticas públicas que promueven la participación infantil. Varios autores (Durán-Strauch, 2017; Figueroa-Grenett, 2018; Gaitán \& Liebel, 2011; López-Contreras, 2015; Trilla \& Novella, 2001) han señalado que no es suficiente con el reconocimiento político y legal de la participación infantil, si esta no se hace práctica; y si, más que un derecho de NNA, se la sigue comprendiendo como una obligación adulta (institucional) o sigue subordinada a las creencias, el lenguaje, los presupuestos morales y el arbitrio de la interpretación adulta que determina el lugar y el papel adecuado de la participación infantil.

Este tipo de significaciones se complejiza con las visiones aportadas por autores que analizan el fenómeno de la participación infantil en relación con los medios y las TIC, pues sus hallazgos añaden nuevas discusiones a los significados y sentidos de la participación de los NNA, que incluyen más géneros o modalidades que rangos o jerarquías. En la esfera mediática y digital, la participación infantil es un campo de estudio emergente y en constante evolución, debido a las transformaciones tecnológicas propias de un contexto de convergencia digital. Las tendencias identificadas analizan la participación infantil desde las prácticas mediáticas, los usos y las características de consumo y acompañamiento familiar, con escasas discusiones epistemológicas y metodológicas de las particularidades que adquiere este fenómeno cuando se estudia desde la perspectiva de las infancias.

Una primera tendencia se ha orientado al análisis de las prácticas participativas de los NNA desde una perspectiva de riesgos y oportunidades (Byrne et al., 2016), así como a la producción cultural con fines lúdicos y de socialización (Ito et al., 2010). En el marco del proyecto Global Kids Online, que reúne una tradición investigativa de más de veinte años, la participación es entendida como una categoría específica en el contexto de las prácticas mediáticas realizadas por los actores infantiles. Esta experiencia de acción supera la connotación cívica y política que demarca las referencias a una participación infantil 
deseada o ideal, así como abre espacios para pensar una participación donde también el ocio y la socialización tienen lugar. En esa misma línea, Ito et al. (2010) analizan la participación desde una perspectiva cultural y social, teniendo en cuenta los contextos mediáticos y las formas de negociación de los jóvenes. Así, se establecen géneros de participación, definidos por los intereses personales y por la amistad, que superan el enfoque hacia niveles o grados de participación donde unos son más sofisticados que otros. Los géneros de participación proponen una visión sobre la diversidad de las prácticas del público infantil y juvenil en relación con formas particulares de socialización e identificación en la oferta mediática.

Otra tendencia de investigación está interesada en analizar las diferentes formas de participación política y cívica. Vizcaíno-Laorga et al. (2019) proponen una clasificación de usos y participación de los jóvenes en las redes sociales que va desde el nivel cero, en el cual los usuarios están en una red por motivos sociales, hasta el mayor nivel, denominado como ciberactivismo, en el que se presentan acciones interactivas con diferentes niveles de intensidad. En una línea similar, Barredo et al. (2018) realizaron un estudio con jóvenes colombianos en el que hacen un trabajo comparativo de consumo de medios en línea y fuera de línea, concluyendo que las y los jóvenes tienen cada vez menor consumo y confianza en los medios convencionales, además de que existe una correlación positiva entre la participación política y el mayor consumo de medios digitales. Diferentes investigaciones también han indagado por los movimientos juveniles de las denominadas generación hashtag y generación blockchain (Feixa, 2021) y han mostrado los usos juveniles de los canales digitales para verificar la información que los adultos y los medios oficiales proporcionan (Kaskazi \& Kitzie, 2021).

Estudios adicionales sobre la participación infantil se han concentrado en el análisis de estas prácticas participativas en el contexto de la convergencia mediática. Así, LafaurieMolina (2012) indaga sobre la respuesta de los NNA frente a las posibilidades de interactividad crossmedia en los canales de televisión infantil, estableciendo modalidades en la oferta interactiva que condicionan las formas de respuesta de los públicos. Woodfall y Zezulkova (2016) analizan las mutaciones en las prácticas mediáticas de los NNA frente a una opción multiplataforma, estableciendo la figura de platform agnostic child como la respuesta de estos para interactuar con una oferta mediática amplia y diversa. LópezOrdosgoitia y Rio (2020) analizan las modalidades de participación en función de los roles mediáticos de los NNA en un contexto de televisión pública multiplataforma. Winocur et al. (2018) indagan las habilidades y estrategias transmedia en adolescentes de 
ocho países, incluyendo Colombia, centradas en el juego, la socialización y la producción creativa como formas de participación formativas en escenarios de aprendizaje informal.

En esa misma línea, se analizan las características de consumo y mediación familiar, donde las estrategias parentales se limitan a establecer control en tiempos y contenidos (Torrecillas et al., 2020) o varían en función del género y la edad, implicando «una situación de desventaja digital» (Martínez et al., 2020, p. 73). Se destaca igualmente el trabajo de Fuente et al. (2019), en el que los autores analizan los procesos de alfabetización mediática de jóvenes en contextos de participación a partir del uso de recursos digitales y la creación de universos transmedia; así como los estudios de Hirsjärvi y Tayie (2011), en los que se argumenta que muchos de los roles y formas de participación de los NNA y sus familias dependen en gran parte de sus características contextuales y sociodemográficas.

Los estudios referenciados plantean preguntas sobre las diferentes formas de participación de los públicos infantiles, así como sobre la aparición de nuevos ámbitos de expectativa que invitan a asumir diferentes roles en los procesos de comunicación. La presencia de un ecosistema mediático complejo, que permite la creación, la recomendación y la acción de compartir contenidos, reconfigura el proceso de comunicación, anteriormente pensado desde una lógica vertical, donde solo tenían cabida espacios simbólicos de participación.

Estas investigaciones destacan, además, la necesidad de seguir indagando el fenómeno desde la experiencia y la perspectiva de sus protagonistas: los niños y las niñas, de modo que sea posible «aprender a partir de las experiencias de los niños y jóvenes de interactuar con las tecnologías digitales y escuchar lo que quieren y esperan de los actores claves que pueden realmente hacer la diferencia a sus vidas digitales» (Mukherjee \& Livingstone, 2020, p. 3).

Así, la participación infantil en el contexto mediático actual es definida no solo por la autonomía de los públicos, sino por su carácter interaccional (Enli, 20o8), estableciendo nuevas formas de implicación para los NNA, permitiéndoles ser actores en la experiencia mediática de la comunicación (Loicq \& Kredens, 2016). La participación se sitúa en una lógica amplia, donde las distintas prácticas de interacción y de creación convergen en un ecosistema mediático multiplataforma que ofrece una amplia variedad de roles a los públicos infantiles, desde el simple visionado hasta procesos de recomendación y creación. Este abanico de posibilidades - que se abre gracias a las tecnologías digitalespermite situar la noción de la participación en cuestiones relacionadas con el equilibrio y el poder en la toma de decisiones (Carpentier, 2012), retomando puntos en común con 
la noción de participación infantil definida por Hart (1993), sin limitar el entendimiento a opciones ideales de la misma.

El presente trabajo contribuye al debate de la participación infantil en el contexto mediático a través de una interpretación sobre las experiencias de participación de un grupo de NNA colombianos entre los 7 y 14 años. El análisis consignado en este artículo ofrece luces respecto a los modelos metodológicos y epistemológicos que acercan la perspectiva infantil a un entendimiento sobre lo que significa participar para los NNA en escenarios digitales, donde las lógicas adultocéntricas que rigen los universos infantiles entran en cuestión. Sabemos que los NNA se encuentran en contacto con las pantallas desde edades cada vez más tempranas (Chaudron, 2015; Unicef, 2017) y que estas ocupan un lugar importante en su cotidianidad. Identificar sus experiencias de participación en los medios puede aportar a hacer tales dinámicas participativas visibles, legitimadas socialmente y despojadas de juicios de validez. De esa forma, se contribuiría a tomar conciencia del valor de las acciones infantiles, de su impacto en los entornos y de la configuración de la categoría de infancia. Todo esto se justifica en la necesidad de facilitar condiciones para el ejercicio de la participación en los medios y el entorno digital; algo que sin lugar a dudas se aprende participando.

El objetivo principal del estudio fue entonces comprender los sentidos de participación presentes en la relación que los NNA establecen con los medios y las TIC. Para ello, se establecieron como objetivos específicos:

1. Identificar las formas de consumo y uso de medios y TIC en los NNA, en relación con el tipo de plataforma, modos de acceso y contenidos.

2. Identificar las nociones y experiencias de participación de los NNA en los medios y las TIC.

3. Interpretar el significado otorgado por NNA a la participación en medios y las TIC a partir de sus intereses, necesidades y expectativas infantiles.

\section{Método}

Este estudio se enmarca en un proyecto de investigación más amplio, que se propone caracterizar la relación de NNA de 7 a 14 años con los medios, desde el acceso, los usos y la participación infantil. El estudio implicó una primera fase cualitativa, en torno a la construcción de una encuesta que sería lanzada en una segunda fase cuantitativa, para lo 
cual se vinculó a un grupo de NNA en calidad de colaboradores en el diseño del instrumento. La fase cualitativa sirvió al mismo tiempo para indagar sobre las perspectivas y experiencias que estos mismos colaboradores compartían en torno a la participación infantil en medios. El presente artículo responde así a este primer momento cualitativo.

El diseño metodológico empleado corresponde a un estudio cualitativo de tipo descriptivo, que permitió indagar, a través de un proceso de colaboración con un número limitado de informantes, la comprensión de un determinado fenómeno social (Vasilachis, 2014). En el ejercicio participaron 75 NNA de tres instituciones educativas y de tres organizaciones locales que atienden población infantil, en las ciudades de Barranquilla, Bogotá y Cali (Colombia). Para su incorporación y selección, inicialmente se contactaron y convocaron diferentes instituciones y organizaciones, de las cuales se eligieron finalmente algunas de entre las que expresaron su deseo voluntario de participar en el proyecto. Logrado esto, se extendió la invitación para que los NNA vinculados a estas instituciones participaran si así lo deseaban, explicando el proyecto y recogiendo luego consentimientos y asentimientos. En cada escenario, se conformó un grupo de trabajo, como se muestra en la tabla 1.

\section{Tabla 1}

\section{Grupos de niños y niñas colaboradores}

\begin{tabular}{lll}
\hline Ciudad & \multicolumn{1}{c}{ Institución educativa (IE) } & \multicolumn{1}{c}{ Organización local (OL) } \\
\hline Barranquilla & Institución educativa privada (IE1) 12 NNA & $\begin{array}{l}\text { Organización pública (biblioteca) (OL1) 14 NNA } \\
\text { Bogotá }\end{array}$ \\
Institución educativa pública (IE2) 20 NNA & $\begin{array}{l}\text { Centro cultural y de divulgación científica público } \\
\text { (club de astrología) (OL2) 3 NNA }\end{array}$ \\
Cali & Institución educativa pública (IE3) 14 NNA & $\begin{array}{l}\text { Organización privada sin ánimo de lucro (corpo- } \\
\text { ración para el desarrollo humano) (OL3) 12 NNA }\end{array}$ \\
\hline
\end{tabular}

Cada contexto brindó la oportunidad de interactuar con un conjunto heterogéneo de NNA. Por un lado, logramos identificar que, de acuerdo con las comunidades y las poblaciones atendidas en cada institución y organización, sus niveles socioeconómicos oscilaban entre todos los estratos (altos, medios y bajos). Por otro lado, si bien todos estaban escolarizados, sus trayectorias escolares o las modalidades de escolarización eran variadas. La mayoría asistía al colegio (cursando grados desde segundo de primaria hasta noveno de bachillerato), pero otros estaban inscritos en homeschooling y algunos se encontraban en aulas de aceleración para nivelación, por extra edad. 
Los participantes intervinieron en talleres investigativos de tres sesiones, en cuyo diseño se buscó propiciar un espacio común entre las investigadoras y los NNA para la problematización de la investigación, la discusión del significado de los medios y las tecnologías, la identificación de sus posibilidades y tipos de participación en la oferta mediática y digital, así como la realización de preguntas que fueran susceptibles de hacer a otros NNA para conocer acerca de la situación expuesta.

Establecer un diálogo intergeneracional acerca de la comprensión infantil sobre aspectos relacionados con los medios y las TIC puede presentar varios desafíos. En este sentido, el uso de métodos visuales, acompañados de otras técnicas como entrevistas y talleres investigativos, facilitó acceder tanto al conocimiento articulado como a la comprensión implícita de los participantes (Owen et al., 2007). Esta combinación de técnicas ofrece la posibilidad de acceder a la comprensión crítica de los NNA, frente a las limitaciones exclusivas de un espacio de preguntas realizadas por las investigadoras, especialmente si son formuladas en un vocabulario pensado por el adulto (López-Ordosgoitia \& Rodrigues, 2014). Con mayor presencia en la literatura anglosajona, los métodos visuales en la investigación social son cada vez más utilizados (Prosser, 1998) y en el trabajo con NNA son reconocidos como una manera de lograr una participación eficaz (James \& Prout, 1997).

La recolección de los datos se llevó a cabo durante la realización de talleres en las seis instituciones/organizaciones mencionadas, entre los meses de octubre y diciembre de 2019, utilizando la técnica graphics elicitation para la obtención de la información, como método visual que potenciara la interlocución con los participantes. Se diseñó entonces un instrumento específico para explorar aspectos cualitativos sobre las modalidades de participación y su experiencia en ese aspecto. Por último, se invitó a la realización de gráficos sencillos, con instrucciones simples, en los que no se precisara de habilidades artísticas para que los niños y las niñas pudieran realizarlos (figura 1).

Cada taller estuvo conformado por tres sesiones (para un total de dieciocho en el conjunto de las tres ciudades), de dos horas de duración cada una, con objetivos y momentos específicos indicados en la tabla 1.

El análisis de los datos se llevó a cabo de manera coordinada entre el equipo de investigadoras de las tres ciudades mediante un proceso en diferentes fases. Primero, se realizó un ejercicio de transcripción y codificación del material recolectado (gráficos, entrevistas, audios, videos) y una primera lectura de la información, codificando las transcripciones, así como la información reportada por los NNA en los instrumentos de métodos visuales. Por tratarse de un conjunto heterogéneo y diverso de datos cualitativos, 
correspondiente tanto a las distintas realidades contextuales como a las subjetividades propias de los NNA, se optó por una codificación inductiva que permitiera un reconocimiento más detallado y menos generalizante de sus testimonios y experiencias significativas en torno al uso y la participación en los medios. Luego, se realizó retroalimentación de los hallazgos obtenidos en las tres ciudades.

Figura 1

Instrumento para explorar los mapas mediáticos

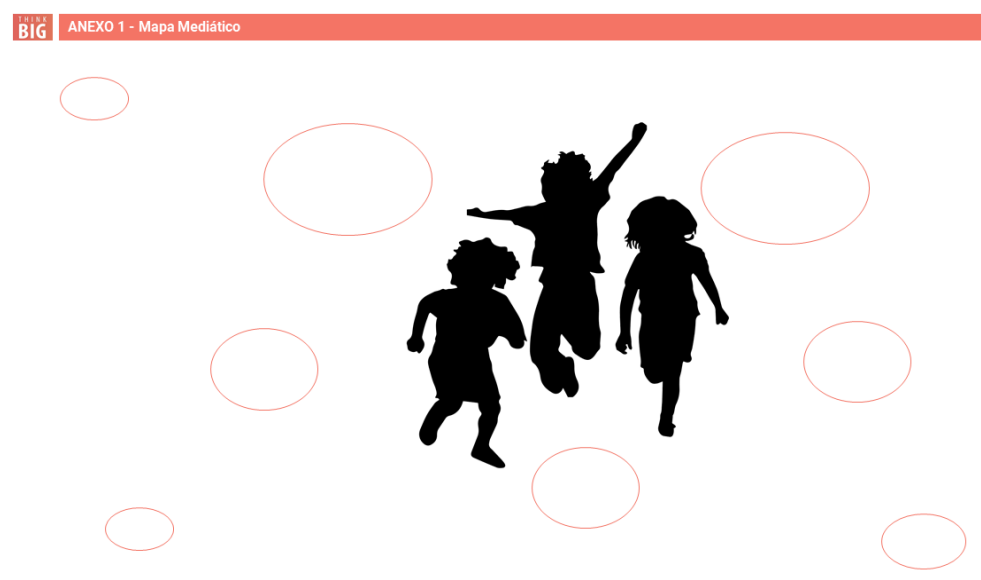

Tabla 2

Diseño de los talleres investigativos

\begin{tabular}{|c|c|c|}
\hline Sesión & Objetivo & Momentos y técnicas \\
\hline 1 & $\begin{array}{l}\text { Identificar las formas de consumo } \\
\text { mediático de los NNA }\end{array}$ & $\begin{array}{l}\text { Momento 1. Mapas mediáticos } \\
\text { Momento 2. Funciones de los medios } \\
\text { Momento 3. Formulación de preguntas }\end{array}$ \\
\hline 2 & $\begin{array}{l}\text { Identificar tanto las nociones de participación } \\
\text { de los NNA como sus experiencias de partici- } \\
\text { pación en los medios }\end{array}$ & $\begin{array}{l}\text { Momento 1. ¿Qué es participar? Conversa- } \\
\text { ción grupal. } \\
\text { Momento 2. Participación en medios. } \\
\text { Momento 3. Formulación de preguntas }\end{array}$ \\
\hline 3 & $\begin{array}{l}\text { Leer las preguntas formuladas por los NNA de } \\
\text { las tres ciudades y seleccionar las que podrían } \\
\text { ir en la encuesta }\end{array}$ & $\begin{array}{l}\text { Momento 1. Revisión de preguntas } \\
\text { Momento 2. Selección de preguntas } \\
\text { Momento 3. Discusión }\end{array}$ \\
\hline
\end{tabular}

La validación de la codificación desarrollada y de las categorías emergentes tuvo como base la congruencia interpretativa en las lecturas hechas por las investigadoras. Por último, se realizó una segunda revisión de los datos obtenidos y se eligieron los hallazgos más representativos para la estructuración del análisis temático, tal como se presenta en el apartado de resultados. 
Los NNA fueron tanto participantes como sujetos de estudio. Tener en cuenta la perspectiva infantil sobre la problemática abordada, así como sobre el proceso mismo de investigación, trazó rutas nuevas e inesperadas para el desarrollo del estudio. Las distintas perspectivas de los NNA sobre los temas tratados en los talleres, especialmente aquellas que de manera espontánea surgían cuando se les preguntaba por su idea de participación, problematizaron algunas de las categorías y conceptos que dábamos por sentados, como se expone en los resultados.

\section{Resultados}

A continuación se presentan los principales resultados de esta investigación, teniendo en cuenta algunas de las categorías emergentes en el proceso de análisis de los datos.

\section{La noción de participación de los niños y las niñas}

Al preguntar a los NNA del estudio por su participación en los medios y las TIC, se observó que no relacionaban fácilmente estos dos elementos. En algunos casos, sus referentes de participación estaban fuertemente anclados en las interacciones cara a cara y esto hacía que les parecieran poco claras algunas de las preguntas que les hacíamos sobre el tema. Se interpreta que las nociones de participación que han construido dependen mayoritariamente de escenarios familiares, escolares y comunitarios, a pesar de que los medios y tecnologías son un espacio importante en sus vidas. En específico, la escuela aparece como el espacio que más ha reforzado, por lo menos desde lo discursivo, la importancia de la participación infantil en acciones concretas, sean estas escolares, recreativas, cívicas, deportivas o artísticas, o en actividades como conversar con otros (hablar, contestar preguntas, opinar, compartir ideas o proponer hacer algo que les guste), asistir a eventos o ir de vacaciones. Tales hallazgos se corresponden con la crítica introducida por determinados autores (Gaitán \& Liebel, 2011; Lay-Lisboa \& Montañes, 2018; LópezContreras, 2015; Trilla \& Novella, 2001) quienes cuestionan si son los adultos los que determinan los roles de la participación infantil, que los NNA van apropiando como significados, discursos y prácticas de la participación.

Investigadora 3: Por acá, ¿cómo participan en los medios?

Niño 29: No sé, ien los medios?

Investigadora $3:$ ujum...

Niño 29: En internet, uno también puede jugar en internet. (Bogotá, taller, IE2) 
Para algunos NNA se hacía difícil identificar dicha participación porque el espectro de posibilidades que ofrecen los medios no encaja claramente en sus definiciones más asentadas de lo que significa participar. Probablemente, les costaba asociar al consumo cultural, al ocio y a la socialización (Ito et al., 2010) con la participación. La naturalidad con la que esas situaciones se dan en sus vidas puede estar distanciada de las nociones estructuradas de participación que han aprendido en otros contextos. Como investigadoras reflexionamos sobre lo que significa reconocer estas dificultades interpretativas, no como impedimentos de comprensión propios de su desarrollo cognitivo, sino como verdaderos interrogantes que se abren en el problema de estudio, así como un indicio de que en la esfera mediática y digital faltan muchos más escenarios y pedagogías de participación infantil.

\section{La participación pasa por el uso de los medios y las TIC}

Al cuestionar de forma puntual a los NNA sobre su participación en los medios y las TIC, aparece una multiplicidad de respuestas en las que relacionan diferentes usos de los medios y tecnologías, y que, además, fueron expresadas con detalle y entusiasmo. Esto se relaciona con una presencia variada, amplia y continua de distintos tipos de contenidos y pantallas, implicando intereses y formas de consumo convergente y transmedial que van desde lo educativo hasta lo lúdico y lo social. Se interpreta que aquello que los NNA entienden por participación en los medios está conectado generalmente con los usos o las actividades que pueden desarrollar a través de estos; aunque no necesariamente ese sentido sea coincidente con el que se relata en la literatura relacionado con el nivel de incidencia, la toma de decisiones y el ejercicio del poder (Carpentier, 2012). Es decir que los NNA tienen sus propias comprensiones de lo que significa participar en los medios, que varían dependiendo del medio y las plataformas, porque las respuestas e interacciones que se tejen son tan amplias y diversas como la misma oferta mediática que se consume, coincidiendo en esto con el análisis de Woodfall y Zezulkova (2016).

Niño 9: ¿Qué tanto puedes participar en los medios?

Niño 8: Bastante, porque hay diferentes medios electrónicos en los que tú puedes participar en todo el mundo, como los celulares, como en las tablets, como en los iPads, como en los computadores, Nintendos.

Niño 9: ¿Cómo puedes participar?

Niño 8: De cualquier manera, porque hay unos que participan en reality shows, otros en conversaciones de WhatsApp, llamadas y los niños en clase ayudando en su casa también 
participan en llamadas a los conocidos. Los niños también participan en algo que quieran participar, por ejemplo, un concurso de talentos para niños o La voz kids y cosas así. (Barranquilla, entrevistas en video, IE1)

Relacionado con lo anterior, se encuentra que los niños y las niñas priorizaron actividades de participación en los siguientes cuatro medios, según lo consignado en sus mapas mediáticos: internet, redes sociales, televisión y videojuegos (en ese mismo orden de importancia). Participar en internet incluye ver vídeos, navegar, buscar información para trabajos o tareas y jugar en línea; aunque también aparecen actividades relacionadas con dar likes, suscribirse a canales de YouTube, responder encuestas, hacer y subir vídeos, opinar y realizar comentarios. En la misma línea, su participación en redes sociales está relacionada con ver vídeos o fotos, buscar anuncios y divertirse, junto a acciones como dar like o me encanta, chatear, conocer personas, socializar, reaccionar a memes y hacer comentarios (figura 2). Y en la televisión, además de ver y oír programas en alta proporción, participan en encuestas y entrevistas, haciendo llamadas, enviando cartas por promociones o concursos y realizando votaciones en realities. En los videojuegos, participar significa jugar y reportar a los jugadores tramposos, es decir, aquellos que usan estrategias engañosas para ganar. Tal multiplicidad de opciones se asocia a los diversos géneros de participación (Ito et al., 2010) que vivencian los NNA dependiendo de sus intereses personales, identidades construidas, formas de socialización y oferta mediática consumida, evidenciando que para ellos no existen categorizaciones tan estructuradas o excluyentes.

\section{Figura 2}

\section{Mapa mediático}

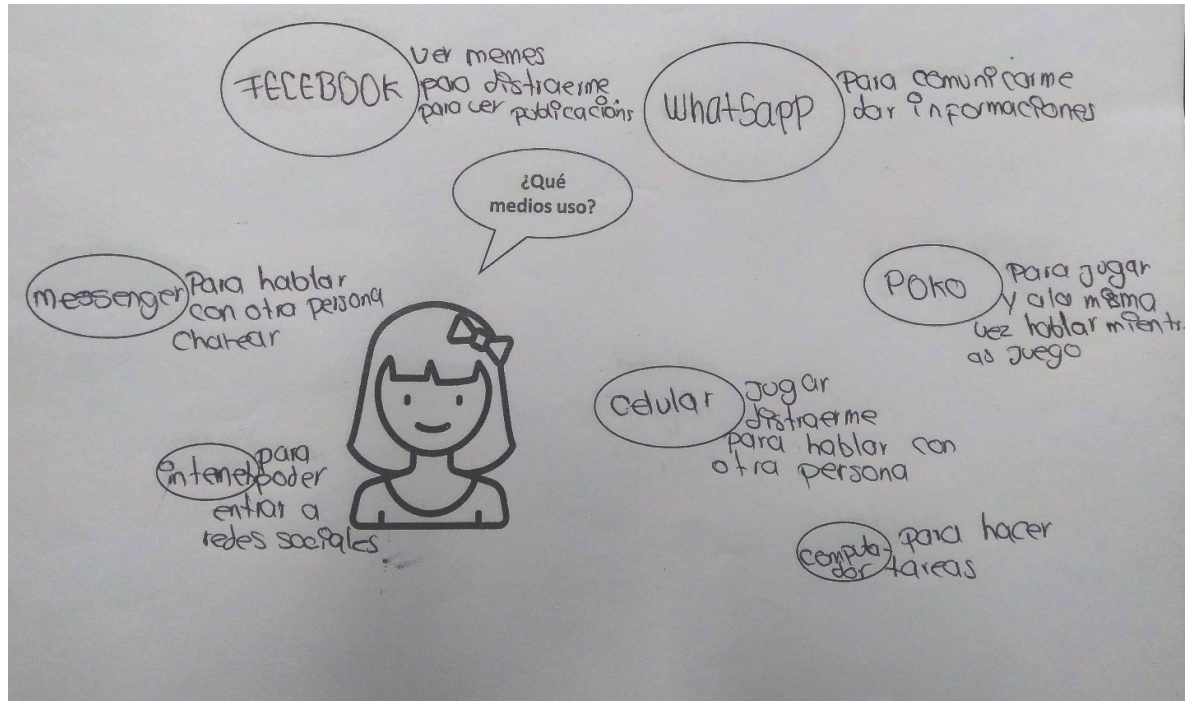


Así, los NNA fueron capaces de complejizar la noción de participación, pues algunos de ellos mencionaron que un mismo medio de comunicación o plataforma podía ofrecerles diferentes modalidades de participación, mientras que con otros tenían mayores dificultades para conocer cómo participar, algo que sucedió particularmente con la prensa y el cine. Por ejemplo, al preguntarles si participaban en la prensa, se obtuvieron respuestas del tipo: «Uno no puede participar»; «No, porque solo participan en la prensa la gente como futbolistas, actores».

Para los NNA, participar en medios significa experiencias diversas, refiriéndose a contenidos variados, distintas plataformas o dispositivos y usos diferenciados. Se muestran diestros en algunos usos, pero también curiosos por conocer lo que sus pares hacen y cómo lo hacen.

\section{Una participación que no siempre es la deseada por los niños, las niñas y adolescentes}

Ahora bien, para algunos NNA las opciones de dar likes, suscribirse o responder a concursos o promociones que ofrecen los medios pueden vivirse como experiencias que no están ausentes de molestias o frustraciones. Lo anterior se relaciona con sentirse engañados o no beneficiados por las recompensas que se ofrecen desde la invitación a participar; es decir, tienen expectativas de obtener beneficios específicos y notan que no siempre las promesas de participación se cumplen, además de que las entienden como estrategias publicitarias y no como intenciones genuinas de considerar su participación. También manifiestan desagrado por el exceso de publicidad y plantean estrategias para evitarla.

Esto es revelador, pues se constata que los NNA, al hacer práctico su ejercicio de participación, estructuran criterios de selección para resistirse a algunas opciones validadas en los medios. Es decir, son capaces de identificar que algunas propuestas de la industria mediática tienen intenciones distintas a las que aparentemente presentan o no se corresponden con sus deseos de participación, pues irrumpen en los usos que se encuentran haciendo de los medios. Tal situación deja en evidencia que las lógicas mercantiles se filtran en los consumos de los NNA, direccionando la relación con los medios a estas dinámicas propias.

\section{La participación que reconocen a través de otros}

Llama la atención que, aunque la radio, la prensa y el cine no son medios de preferencia para NNA, resultan respuestas coincidentes con actividades que pueden considerarse 
como participación en los mismos, desde llamar a las emisoras de radio a solicitar una canción, llenar los juegos de los periódicos, actuar (relacionado con cine y teatro), votar por la calidad de las películas de cine o dejar comentarios de las mismas, hasta leer noticias, escuchar música o chistes y ver películas. Se interpreta que las referencias a estos medios pueden provenir de escasas experiencias con ellos o de la identificación de formas de participación según el tipo de medio, aunque no necesariamente las hayan realizado personalmente. Se asume que pueden tener conocimiento de que otras personas cercanas lo hacen y por eso las conocen.

Por otro lado, al ser cuestionados sobre si la participación en medios y TIC es un asunto de niños o de adultos, se encuentran respuestas divergentes en dos sentidos: o es preferentemente algo de adultos, porque las actividades que se allí realizan como participación no son de niños, o es un asunto de cualquier persona (niño o adulto). Ambas tendencias muestran la pertinencia de los aportes de Liebel y Saadi (2012), cuando señalan la importancia de reconocer el carácter relacional de la participación entre NNA y adultos, dependiendo de la particularidad de los contextos y los roles otorgados a la infancia en ellos.

\section{La participación que interesa a los niños, las niñas y adolescentes}

Los NNA manifiestan interés por actividades de creación de contenidos que puedan circular a través de plataformas digitales y redes sociales. De manera especial, hay varias referencias de su gusto por la creación de memes y stickers:

Investigadora 3: ¿Han hecho memes?

Varios niños: Ah, eso sí... [ríen]

Investigadora 3: [ríe] ¿Sí?, ¿cómo han hecho memes?

Niño 31: Sí, con foto de los amigos.

Niña 33: Sí, con foto de todos los amigos, sí.

Investigadora 3: ¿Sí? ¿Y lo ponen a circular en qué, en WhatsApp o en redes?

Niño 44: No, entre nosotros mismos, se hacen stickers.

Investigadora 3: ¿Y cómo se hace lo de los memes?

Niño 37: Eh... con páginas.

Niño 45: No, yo solo tomo un pantallazo y le coloco texto abajo y ya; la fácil.

(Bogotá, taller, IE2) 
Además, varios manifiestan su deseo por ser youtubers y así colaborar con sus seguidores, a través de la generación de contenidos que consideran de utilidad. Otros mencionan experiencias en compañía de hermanos, primos o tíos, en las que hacen vídeos en redes sociales, como por ejemplo, directos de Instagram. Relacionado con ello, algunos NNA mencionan que, gracias a los comentarios o reacciones que hacen a los influencers, estos generan nuevos contenidos siguiendo lo que les dicen. Ello representa una conciencia acerca de cómo se producen algunas dinámicas de comunicación y participación, en las que los públicos tienen algún tipo de incidencia sobre lo que sucede en la producción de contenidos.

\section{La participación como actividad mediada por adultos}

Varios de los NNA aluden a su participación en los medios tras el conocimiento y acompañamiento de sus padres, madres o cuidadores. En ese sentido, la participación infantil parece estar preferentemente mediada por los adultos. Los NNA reconocen que muchas veces sus posibilidades de participación deben estar autorizadas por los maestros, padres, cuidadores, en espacios seleccionados y conducidos por estos. Así, más que un derecho, en ocasiones «sigue dependiendo de la voluntad y las decisiones de los adultos» (Liebel \& Markowska-Manista, 2021, p. 1), cuando ellos los dejan hablar, cuando les piden la palabra y cuando les dan los espacios.

Investigadora 4: ¿Ustedes creen que los adultos dejamos que los niños participen? Niño 55: Algunos. Mi papá a veces no me deja participar en cosas. Antes no me quería dejar participar en esto.

Niño 51: A veces uno quiere hacer algo en la vida y los papás no lo dejan.

Niño 57: A veces dudan de algo.

Investigadora 4: ¿Qué deberíamos hacer los adultos para dejar participar más a los niños?

Niña 54: Escuchar, tratar de entender.

Investigadora $4: \mathrm{mmm} . .$. , los adultos no entendemos a los niños...

Niño 62: A veces no. (Cali, taller, IE3)

También, muchos distinguen entre los adultos que los dejan participar y los que no; y comprenden que su condición como NNA los sitúa en una doble condición: a veces pensados como sujetos de participación y otras veces como sujetos de cuidado. 
Niña 16: Puedes participar, pero de manera que los papás lo estén vigilando porque de pronto hay muchas personas que son malas y se hacen pasar por otras personas y nos pueden robar, violar y otras cosas más. (Barranquilla, taller, OL1)

Los testimonios evidencian que, en algunos casos, las mediaciones adultas se desdibujan, pues las mismas lógicas de los medios y las TIC así lo permiten. Los NNA buscan sortear las limitaciones o prohibiciones establecidas en sus familias alrededor de la regulación con los medios y las TIC, lo que tal vez en otros espacios no sería posible. Por ejemplo, uno de los niños participantes de la organización local de Bogotá nos mencionó que, frente a las restricciones que tenía en casa para usar el celular, utilizaba un pretexto para lograrlo.

Niño 47: Yo le digo a mi mamá que voy a ver gatos y ella me lo presta. Investigadora $3: ¿ Y$ por qué gatos?

Niño 47: Porque a ella le gustan los gatos y entonces sí me lo presta.

Tal como lo plantean Livingstone y Bober (2006), es una especie de juego familiar: mientras emergen estrategias parentales para moderar el uso que los NNA hacen de los medios y las TIC, también estos desarrollan tácticas para evadir o resistir, teniendo generalmente mayor confianza y experticia en los medios digitales que sus padres.

\section{Discusión}

La investigación con los NNA participantes reveló diferentes elementos sobre la participación desde la perspectiva infantil. Se trata de una participación convergente y multiplataforma, que no es homogénea y no tiene los mismos significados para los NNA. Dado que sus nociones de participación están estrechamente vinculadas con acciones que dependen de algunos usos de medios y TIC, existen diversas manifestaciones que ellos relacionan como opciones de participación. Ahora bien, en el contexto de un ecosistema mediático complejo, los niños reconocen que en algunos medios y plataformas tienen mayores posibilidades de participación que otros. Por todo lo anterior, la investigación nos permitió comprender que los protagonistas no hacen distinciones sobre la validez o la importancia que tiene un tipo de participación sobre otra, lo que sí suele demarcarse en la literatura de la participación infantil a través de las escalas y categorizaciones de tipos de participación ideales o figurativos. La interpretación escalada en términos 
progresivos y teleológicos sobre la participación mediática, que está muy presente en la literatura, parece no tener para los NNA esta misma rigidez y carga valorativa, que clasifica y presenta un tipo de participación mediática como mejor que otra. Dar like en un video o una publicación puede ser tan significativo para los NNA como opinar y realizar comentarios, llamar para pedir una canción en una emisora o producir contenidos para las redes sociales. Igual ocurre con su valoración sobre la participación mediática con fines escolares, de ocio o de socialización. Así, desde las respuestas de los NNA involucrados en la investigación, participar en los medios y las TIC incluía acciones, dispositivos, escenarios mediáticos y formas de interacción que eran valoradas por lo que les permitían hacer, sentir y relacionarse. En términos de goce, disfrute y humor, esto representaba para ellos lo que podían lograr (estímulos, premios, reconocimiento de pares) y qué tan visibles podían ser sus acciones de participación.

La interacción infantil con los medios y las TIC se plantea, en su mayoría, desde los proyectos y expectativas que se han trazado los adultos e instituciones sobre el tema. Por ello se presentan divisiones, en ocasiones artificiales, sobre lo que significa la participación infantil adecuada, correcta, seria, deseable y significativa versus la que resultaría supuestamente secundaria, ociosa, menos representativa o que no responde a las expectativas de los adultos. En este estudio, notamos que los medios y las TIC representan para los niños, niñas y adolescentes un escenario con lógicas particulares, en el que se resignifican sus relaciones con otros, incluidos los adultos y, por tanto, sus experiencias de participación toman otros matices. Esto nos conduce a insistir en la necesidad de enriquecer un espacio de conocimiento emergente que resulta de la intersección entre las nociones de participación, infancias y ecosistemas mediáticos y digitales.

Es posible pensar que uno de los efectos de las clasificaciones y expectativas que se han construido socialmente sobre lo que debe ser la participación infantil en los medios y las TIC (y, en general, en diferentes ámbitos sociales) es que para los mismos NNA todavía resulta complejo comprender qué posibilidades y espacios de participación tienen. Tal como lo mencionaron varios de ellos, se piensa más como un estatus otorgado, facilitado o mediado por los adultos, que un derecho en sí mismo. De esto también se deriva que falta implementar acciones pedagógicas y espacios de reflexión sobre cómo potenciar la participación infantil en la esfera mediática y digital, así como identificar los riesgos asociados a ella, de manera que la relación de NNA con los medios sea enriquecida y más segura, orientada a fortalecer sus competencias en el ejercicio de la ciudadanía, los derechos y la alfabetización digital (Pangrazio \& Sefton-Green, 2021). 
Este estudio se concentró de manera particular en las experiencias y perspectivas de un grupo de NNA de tres ciudades colombianas sobre la participación infantil en los medios y las TIC: se trata entonces de unas impresiones que también responden a determinadas posibilidades de acceso a medios y tecnologías, sin ignorar que en otras ciudades y municipios rurales o de frontera esta experiencia sea diferente. Por último, también falta nutrir estos resultados con las percepciones de adultos, cuidadores y maestros sobre el mismo tema, así como de productores y creadores de contenido infantil. Es importante pensar la participación infantil como un asunto relacional y no como deber o favor adulto, integrando una perspectiva de corresponsabilidad intergeneracional e intersectorial. En ese sentido, se refuerza la necesidad advertida por Mukherjee y Livingstone (2020) de estudiar la participación infantil en medios desde la propia experiencia de los NNA, sin desconocer las dinámicas específicas de los textos mediáticos y las lógicas internas, tanto de los medios como de las TIC.

\section{Agradecimientos}

Agradecemos de manera especial a los niños, niñas y adolescentes de cada una de las organizaciones locales e instituciones educativas que participaron del estudio.

\section{Referencias}

Barredo, D., de la Garza, D., \& Días, D. (2018). La relación entre el consumo de medios digitales, la participación y la eficacia política: un estudio sobre los jóvenes universitarios en Colombia. Revista Latina de Comunicación Social, 73, 945-960. https:// doi.org/10.4185/rlcs-2018-1290

Byrne, J., Kardefelt-Winther, D., Livingstone, S., \& Stoilova, M. (2016). Global kids online: Research synthesis, 2015-2016. Unicef Office of Research Innocenti; London School of Economics and Political Science. https://bit.ly/zacDVen

Carpentier, N. (2012). The concept of participation: If they have access and interact, do they really participate? Fronteiras-Estudos Midiáticos, 14(2), 164-177. https://doi.org/g7zk

Chaudron, S. (2015). Young children (o-8) and digital technology: A qualitative exploratory study across seven countries. Institute for the Protection and Security of the Citizen (Joint Research Centre). https://bit.ly/3ocFBu7 
Cornwall, A. (2008). Unpacking 'participation': Models, meanings and practices. Community Development Journal, 43(3), 269-283. https://doi.org/10.1093/cdj/bsno10

Cuevas-Parra, P. (2021). Thirty years after the UNCRC: Children and young people's participation continues to struggle in a COVID-19 world. Journal of Social Welfare and Family Law, 43(1), 81-98. http://doi.org/10.1080/09649069.2021.1876309

Durán-Strauch, E. (2017). Derechos de niños y niñas: del discurso a la política local. Revista Latinoamericana de Ciencias Sociales, Niñez y Juventud, 15(2), 879-891.

Enli, G. (2008). Redefining public service broadcasting: Multi-platform participation. Convergence. The International Journal of Research into New Media Technologies, 14(1), 105-120. https://doi.org/10.1177/1354856507084422

Feixa, C. (2021). Generación blockchain: movimientos juveniles en la era de la web semántica. Revista Latinoamericana de Ciencias Sociales, Niñez y Juventud, 19(1), 1-20. https://dx.doi.org/10.1160o/rlcsnj.19.1.4584

Figueroa-Grenett, C. (2018). La acción política de niños, niñas y jóvenes en Chile: cuerpos, performatividad y producción de subjetividad. Revista Latinoamericana de Ciencias Sociales, Niñez y Juventud, 16(1), 199-212. https://doi.org/g7zm

Fuente, J., Lacasa, P., \& Martínez, R. (2019). Adolescentes, redes sociales y universos transmedia: la alfabetización mediática en contextos participativos. Revista Latina de Comunicación Social, 74, 172-196. https://doi.org/10.4185/rlcs-2019-1326

Gaitán, L., \& Liebel, M. (2011) Ciudadanía y derechos de participación de los niños. Universidad Pontificia Comillas; Síntesis.

Hart, R. (1993). La participación de los niños: de la participación simbólica a la participación auténtica. Unicef. https://bit.ly/3mLpXTY

Hirsjärvi, I, \& Tayie, S. (2011). Niños y nuevos medios: estudios de caso en Egipto y Finlandia. Comunicar. Revista Científica de Educomunicación, 19 (37), 99-108. https:// doi.org/10.3916/C37-2011-03-01

Ito, M., Baumer, S., Boyd, D., Cody, R., Herr, B., Horst, H., Lange, P., Mahendran, D., Martínez, K., Pascoe, C. J., Perkel, D., Robinson, L., Sims, C., \& Tripp, L. (2010). Hanging out, messing around, and geeking out: Kids living and learning with new media. The MIT Press. https://doi.org/10.7551/mitpress/8402.001.0001

James, A., \& Prout, A. (1997). Constructing and reconstructing childhood: Contemporary issues in the Sociological study of childhood. Falmer Press.

Kaskazi, A., \& Kitzie, V. (2021). Engagement at the margins: Investigating how marginalized teens use digital media for political participation. New Media \& Society. https://oi.org/10.1177/14614448211009460 
Kay, E., Tisdall, M., \& Cuevas-Parra, P. (2021) Beyond the familiar challenges for children and young people's participation rights: The potential of activism. The International Journal of Human Rights, 1-19. https://doi.org/10.1080/13642987.2021.1968377

Lafaurie-Molina, A. (2012). Interactividad en la oferta de contenidos infantiles cross-media para TDT e Internet: el caso de Superz. [Tesis doctoral no publicada]. Universidad Autónoma de Barcelona.

Lay-Lisboa, S., \& Montañes, M. (2018). De la participación adultocéntrica a la disidente: la otra participación infantil. Psicoperspectivas: Individuo y Sociedad, $17(2), 1-12$. https://doi.org/10.5027/psicoperspectivas-Vol17-Issue2-fulltext-1176

Liebel, M. (2007). Paternalismo, participación y protagonismo infantil. En Y. Corona, \& M. E. Linares (Coords.), Participación infantil y juvenil en América Latina (pp. 113-146). Universidad Autónoma Metropolitana.

Liebel, M., \& Markowska-Manista, U. (2021). Presentación. Cuestiones éticas y epistemológicas en la investigación con niños. Sociedad e Infancias, 5 (especial), 1-4. https:// doi.org/10.5209/soci.74230

Liebel, M., \& Saadi, E. (2012). La participación infantil ante el desafío de la diversidad cultural. Desacatos, 39, 123-140. https://doi.org/10.29340/39.244

Livingstone, D., \& Bober, M. (2006). Regulating the internet at home: Contrasting the perspectives of children and parents. En D. Buckingham, \& R. Willett (Eds.), Digital generations: Children, young people and new media (pp. 93-113). Lawrence Erlbaum.

Loicq, M., \& Kredens, E. (2016). Les plateformes numériques des chaînes de télévision: du fantasme technologique à la réalité des pratiques des jeunes. Les Enjeux de l'Information et de la Communication, 17 (3A), 167-178.

López-Contreras, R. (2015). Interés superior de los niños y niñas: definición y contenido. Revista Latinoamericana de Ciencias Sociales, Niñez y Juventud, 13(1), 51-70.

López-Ordosgoitia, R., \& Rio, F. (2020). Les rôles des jeunes spectateurs dans les stratégies multi-plates-formes. Télévision, (11), 177-195. https://doi.org/10.3917/telev.011.0177 López-Ordosgoitia, R., \& Rodrigues, A. (2014). Los niños y el internet: la nueva sociología de la infancia y los métodos visuales. Revista Comunicando, 3(1), 220-233.

Lundy, L. (2018). In defence of tokenism? Implementing children's right to participate in collective decision-making. Childhood, 25(3), 340-354. https://doi.org/ghx82k

Martínez, G., Casado, M., \& Garmendia, C. (2020). Estrategias de mediación parental en España: factores predictores para los diversos tipos de estrategias. ZER: Revista de Estudios de Comunicación, 20(39), 13-27. https://doi.org/10.1387/zer.15513 
Mukherjee, S., \& Livingstone, S. (2020). Children and young people's voices. Digital Futures Commission; 5 Rights Foundation. https://bit.ly/32lMUnb

ONU. (1989). Convención de los Derechos del Niño. Unicef. https://bit.ly/3uNKZnO

Owen, L., Auty, S., Lewis, C., \& Berridge, D. (2007). Children's understanding of advertising: An investigation using verbal and pictorially cued methods. Infant and Child Development, 16(6), 617-628. https://doi.org/10.1002/icd.535

Pangrazio, L., \& Sefton-Green, J. (2021). Digital rights, digital citizenship and digital literacy: What's the difference? Journal of New Approaches in Educational Research, 10(1), 15-27. https://doi.org/10.7821/naer.2021.1.616

Prosser, J. (ed.) (1998). Image-based research. Falmer Press.

Salazar-Guizzo B., Balduzzi, L., \& Lazzari, A. (2019) Protagonismo infantil: um estudo no contexto de instituições dedicadas à educação da primeira infầncia em Bolonha. Educar em Revista, 35(74), 271-289. https://doi.org/10.1590/0104-4060.64245

Soares Gouvêa, M. C., Carvalho, L., de Freitas, F., \& Bizzotto, L. (2019). O protagonismo infantil no interior de movimentos sociais contemporâneos no Brasil. Sociedad e Infancias, 3, 21-63. https://doi.org/10.5209/soci.63525

Torrecillas, T., Vázquez, T., Suárez, R., \& Fernández, L. M. (2020). El papel de los padres en el comportamiento online de menores hiperconectados. Revista Latina de Comunicación Social, (75), 121-148. https://doi.org/10.4185/rlcs-2020-1419

Trilla, J., \& Novella, A. (2001). Educación y participación social de la infancia. Revista Iberoamericana de Educación, 26, 137-164. https://doi.org/10.35362/rie260982

Unicef. (2017). El estado mundial de la infancia 2017: niños en un mundo digital. Unicef. https://www.unicef.org/media/48611/file

Vasilachis, I. (coord.) (2014). Estrategias de investigación cualitativa. Gedisa.

Vizcaíno-Laorga, R., Catalina-García, B., \& López de Ayala-López, M. C. (2019). Participación y compromiso de los jóvenes en el entorno digital: usos de las redes sociales y percepción de sus consecuencias. Revista Latina de Comunicación Social, 74, 554-572. https://doi.org/10.4185/rles-2019-1345

Winocur, R., Gutiérrez, G., \& Barreneche, C. (2018). Habilidades transmedia de los adolescentes y desafíos pedagógicos. Educación y Ciudad, 35, 169-178.

Woodfall, A., \& Zezulkova, M. (2016). What 'children' experience and 'adults' may overlook: Phenomenological approaches to media practice, education and research. Journal of Children and Media, 10(1), 98-106. https://doi.org/10.1080/17482798.2015.1121889 